www.jmscr.igmpublication.org

Index Copernicus Value: 79.54

ISSN (e)-2347-176x ISSN (p) 2455-0450

crossrefDOI: https://dx.doi.org/10.18535/jmscr/v7i2.18

Journal Of Medical Science And Clinical Research

IGM Publication

An Official Publication of IGM Publication

\title{
Pedunculated submucosal lipoleiomyoma projecting into endocervical canal
}

Authors

\section{Baby Manoj ${ }^{1}$, Shahul Hameed D.K ${ }^{2}$, Innisai A ${ }^{3}$, Laya Attokaran ${ }^{4}$, Sherinas Rehiman}

${ }^{1,3,4,5}$ Consultant Radiologist. Department of Radiology and Imaging, IQRAA International Hospital and

Research Centre, Malaparamba, Kozhikode, Kerala, India

${ }^{2}$ Head of Department. Department of Radiology and Imaging, IQRAA International Hospital and Research

Centre, Malaparamba, Kozhikode, Kerala, India

Corresponding Author

Shahul Hameed D K

Head of Department, Department of Radiology and Imaging, IQRAA International Hospital and Research

Centre, Kozhikode, Kerala,India-673 009

Email:shahuldr@gmail.com

\begin{abstract}
Lipoleiomyoma of the uterus is a rare benign uterine tumour -a variation of leiomyoma ${ }^{[1]}$. The presence of fatty tissue in the myometrium is anomalous, interpreted as lipomatous degeneration, smooth muscle metaplasia or as a benign tumour called as lipoleiomyoma. Imaging plays an important role in determining the intrauterine location and fatty nature of lipoleiomyomas.

We report a rare case of pedunculated submucosal lipoleiomyoma projecting into endocervical canal in which USG and MRI were useful to characterise the lesion, confirmed histopathologically.

Keywords: lipoleiomyoma, uterus, pedunculated, submucosal, myometrium.
\end{abstract}

\section{Introduction}

Lipomatous uterine tumors of uterus are unusual benign neoplasms occurring primarily in obese perimenopausal and post menopausal women. Histologically, these tumors comprise a spectrum including pure lipomas, lipoleiomyomas and fibrolipomyomas. They are composed of smooth muscle and mature adipose tissue.

56 year old postmenopausal woman presented with intermittent lower abdominal pain. USG showed well defined echogenic mass with posterior acoustic attenuation in the lower endometrial cavity and endocervix. Doppler showed vascular pedicle extending from the uterine body. MRI pelvis showed heterointense polypoidal lesion extending into the endocervical canal. Signal loss noted in fat sat sequences. Signal loss noted in out phase images. Hysterectomy done and pathology proved to be lipoleiomyomatous polyp.

\section{Case Report}

56 year old post menopausal woman presented with intermittent lower abdominal pain. No bleeding PV. USG showed well defined echogenic mass with posterior acoustic attenuation in the lower endometrial cavity and endocervix -Fig. 1. Doppler showed vascular pedicle is seen extending from the uterine body. No demonstrable vasularity in the mass-Fig 1. 


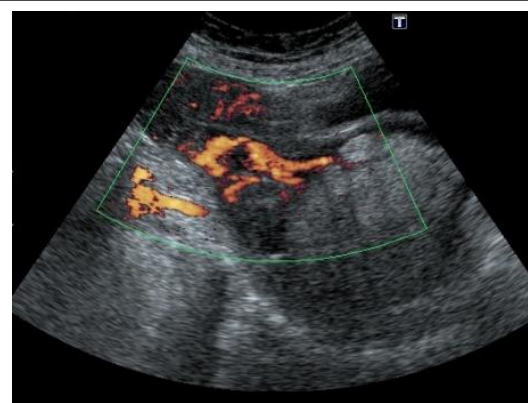

Figure 1 USG and doppler: Echogenic mass in the lower endometrial cavity and endocervix with vascular pedicle extending from the uterine body. MRI pelvis showed heterointense polypoidal lesion extending into the endocervical canal from uterine body. Intramural fibroid in the utero-cervix region posteriorly. T1 weighted images showed hyperintense foci within the lesion -Fig 2. Signal loss noted in fat sat sequences- Fig 3. Signal loss noted in out phase images -Fig 4,5.

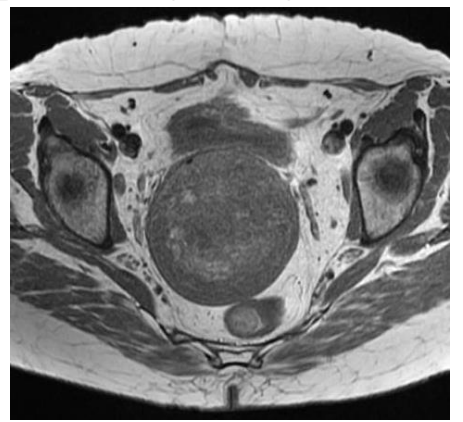

Fig 2-Ax T1 weighted images showed hyperintense foci within the lesion.

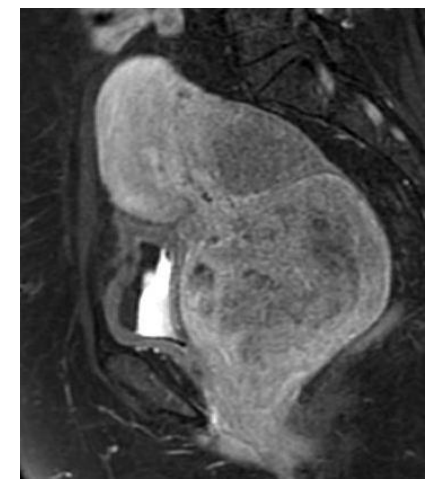

Fig 3- Sag T1 fs- Signal loss in fat sat sequences

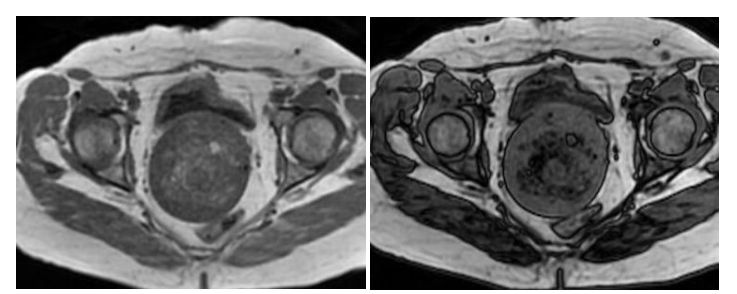

Fig 4,5-Ax- in \& out phase images -Signal loss in out phase images.

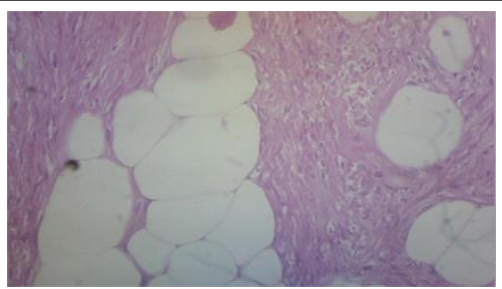

Fig 6 Photomicrograph-adipose cells with spindle cells

Diagnosis of pedunculated submucosal lipoleiomyoma projecting into endocervical canal was made. Hysterectomy was performed and sent for histopathology. Grossly, on cutting open the uterine cavity a well circumscribed yellow mass was present in the submucosal region of uterus projecting into the endocervical canal. Microscopically, the tumour was composed of spindle cells with moderate amount of cytoplasm. Clusters of adipocytes in between the fasciclesFig.6 -Lipoleiomyomatous polyp.

\section{Discussion}

The incidence $0.03 \%$ to $0.2 \%{ }^{[2],[3]}$. Lipoleiomyomas of the uterus are typically found in postmenopausal women. Most of the patients are asymptomatic. If symptomatic, they are similar to that of leiomyoma, i.e., palpable mass, urinary frequency, and bleeding PV. Lipoleiomyomas have also been reported in cervix, broad ligament, retroperitoneum and ovary [4],[5]. Rajeev et al has reported submucosal lipoleiomyoma with pathological findings ${ }^{[6]}$. The pathogenesis of the lesion remains unknown. Several theories have been proposed, including metaplasia of smooth muscle cells or connective tissue into adipose cells (most widely accepted); misplaced embryonic fat cells, lipocytic differentiation of primitive connective or mesenchymal tissue; perivascular extension of peritoneal or retroperitoneal fat.

In ultrasound, these lesions are echogenic with posterior acoustic attenuation. It is partially encased by hypoechoic rim- thought to represent a layer of myometrium surrounding the fatty component ${ }^{[7],[8]}$. Often poor vascularity on doppler. CT is more specific than ultrasound since CT can clearly demonstrate fat component, which appears low in attenuation (HU -40 and -100). Well-marginated 
fat-containing mass arising from uterus, often containing areas of soft tissue density. CT has its own disadvantages, including inability to illustrate detailed uterine anatomy, confusion between an adnexal mass and an exophytic or pedunculated lesion arising from the uterus. MRI- most useful modality in demonstrating the organ of origin because of its multiplanar capabilities. Best tool for diagnosing a lipomatous tumor. Fatty component is high in signal intensity on both $\mathrm{T} 1-$ and T2weighted images. Chemical shift artifacts along the frequency encoding axis may be seen. Fat suppression sequence- demonstrates decrease in signal intensity in fat components of the lesion $^{[8],[9],[10]}$. MRI gives better tissue characterization than CT. Septa inside the mass can be seen on T1-weighted images. Peripheral low signal intensity rim which corresponds to a thin fibrous pseudocapsule. Lipoleiomyoma demonstrates heterogeneous signal intensity with fat and non-fat soft tissue content.

\section{Conclusion}

Combination of clinical history, physical examination and imaging features will probably provide the accurate diagnosis in the majority of cases. MRI characterises the lesion and helps to confirm the diagnosis.

\section{References}

1. Silverberg SG, Kurman RJ. Tumors of the uterine corpus and gestational trophoblastic disease. Washington, DC: Armed Forces Institute of Pathology, 1992:127-130.

2. Brandfass RT, Everts-Suarez EA (1955) Lipomatous tumors of the uterus: a review of the world literature with report of a case of true lipoma. Am j Obstet Gynecol70:359367.

3. Kitajima K, Kaji Y, Imanaka K, Sugihara R, Sugimura K (2007) MRI findings of uterine lipoleiomyoma correlated with pathological findings. AJR Am J Roentgenol 189: 100104.
4. Pounder DJ (1982) Fatty tumours of the uterus. J Clin Pathol 35: 1380-1383.

5. Bajaj P, Kumar G, Agarwal K (2004) Lipoleiomyoma of broad ligament: A case report. Indian J Pathol Microbiol 3: 457-458.

6. Rajeev S, Sumiti G, Shruti S, Ashima Batra, Manish Chaudhary. International J. of Healthcare and Biomedical Research, Volume: 2, Issue: 4, July 2014, Pages 77-79.

7. Prieto A, Crespo C, Pardo A, Docal I, Calzada J, Alonso P. Uterine lipoleiomyomas: US and CT findings. Abdom Imaging 2000; 25:655-657

8. Dodd GD III, Budzik RF. Lipomatous uterine tumors: diagnosis by ultrasound, CT, and MR. J Comput Assist Tomogr 1990; 14:629-632.

9. Aizenstein R, Wilbur AC, Aizenstein S. CT and MRI of uterine lipoleiomyoma. Gynecol Oncol 1991; 40:274-276.

10. Ishigami $\mathrm{K}$, Yoshimitsu $\mathrm{K}$, Honda $\mathrm{H}$, et al. Uterine lipoleiomyoma: MRI appearances. Abdom Imaging 1998; 23:214-216. 